\title{
Overview Percutaneous Nephrolithotomy - A Case Report 32-Year-Old Male with Renal Calculi
}

\author{
Ayun Cassell III, Mohamed Jalloh, Abdurama Diallo, Issa Labou, Lamine Niang, Serigne Gueye \\ Department of Urology and Andrology, General Hospital of Grand Yoff, Dakar, Sénégal \\ Email address: \\ ayuncasselliii@gmail.com (Ayun C. III) \\ To cite this article: \\ Ayun Cassell III, Mohamed Jalloh, Abdurama Diallo, Issa Labou, Lamine Niang, Serigne Gueye. Overview Percutaneous Nephrolithotomy - \\ A Case Report 32-Year-Old Male with Renal Calculi. International Journal of Clinical Urology. Vol. 3, No. 1, 2019 , pp. 6-9. \\ doi: $10.11648 /$ j.ijcu.20190301.12
}

Received: January 6, 2019; Accepted: January 29, 2019; Published: February 19, 2019

\begin{abstract}
Surgical management of renal tract stone disease has evolved during the last two decades after the introduction of minimal invasive techniques. Percutaneous nephrolithotomy (PCNL) has become a common procedure performed in patients with renal calculi. Nevertheless, the learning curve is steep and most facilities in subsaharan African lacks this novel minimally invasive approach. Therefore, the PCNL procedure is clearly illustrated in this review using a Case Report of a 32 year old Mauritania Male with a $2 \mathrm{~cm}$ renal calculi. With standardized technique and the proper equipment, PCNL can achieve excellent stone-free rates and with minimal patient morbidity.
\end{abstract}

Keywords: Calculi, Nephroscope, Percutaneous Nephrolithotomy, Ureteroscope

\section{Introduction}

Management of patients suffering from urinary tract calculi is a health care problem because of its prevalence and recurrence [1]. Minimally invasive treatments, such as percutaneous nephrolithotomy (PCNL), non-invasive extracorporeal shock wave lithotripsy (ESWL), and laparoscopy procedures have almost completely replaced open surgery in the management of the urinary stone disease $[2,3]$.

Recently European Association has considered PCNL as first option for large, multiple or inferior calyx stones [1]. Open stone surgery has been replaced by PCNL because of its cost effectiveness, lower morbidity, shorter operative time and lower postoperative complications $[1,4]$. Some patients with history of open stone surgery need PCNL because of renal stone recurrences $[1,5,6]$.

The learning curve for PCNL is very steep and endourology in Subsaharan Africa is still evolving. Most Urologist in in the tropics are yet to perform their first procedure. The African Literature on PCNL is also sparse. Therefore, this case report of Percutaneous Nephrolithomy highlights the procedure and techniques of PCNL for educative purpose with original illustration during the procedure.

\section{Case Report}

A 26 year old Mauritanian male was seen through the outpatient clinic with colicky right lumbar pain radiating to the groin over a year. He had an open surgery for a Pelvic Ureteral Junction Obstruction 2 years earlier. His Physical Exam was unremarkable. Hematological assays were normal and urine culture sterile. Unehanced computed tomogaphy showed a right pelvicalyceal stone measuring two centimeters in diameter with grade II ipsilateral hydronephrosis. Hematological assays were normal and urine culture sterile. He underwent a percutaneous Nephrolithomy with a ureteral catheter and a nephrostomy tube in place. There was no postoperative bleeding or infection. The patient was discharged home on postoperative day 3 . His subsequent follow-ups have been satisfactory. The operative procedure is illustrated below. 


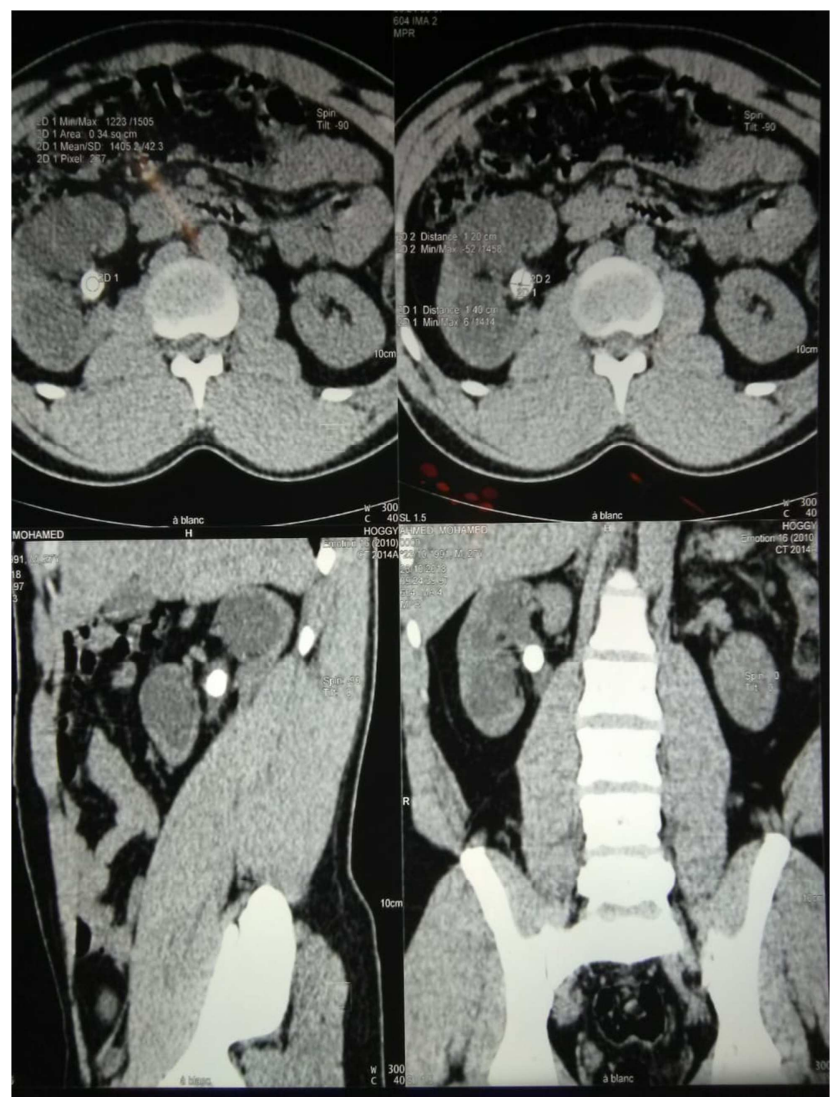

Figure 1. Shows a 2 centimeter right pelvicalyceal with grade III hydronephrosis but the contralateral left kidney has a normal renal parenchma without stone.

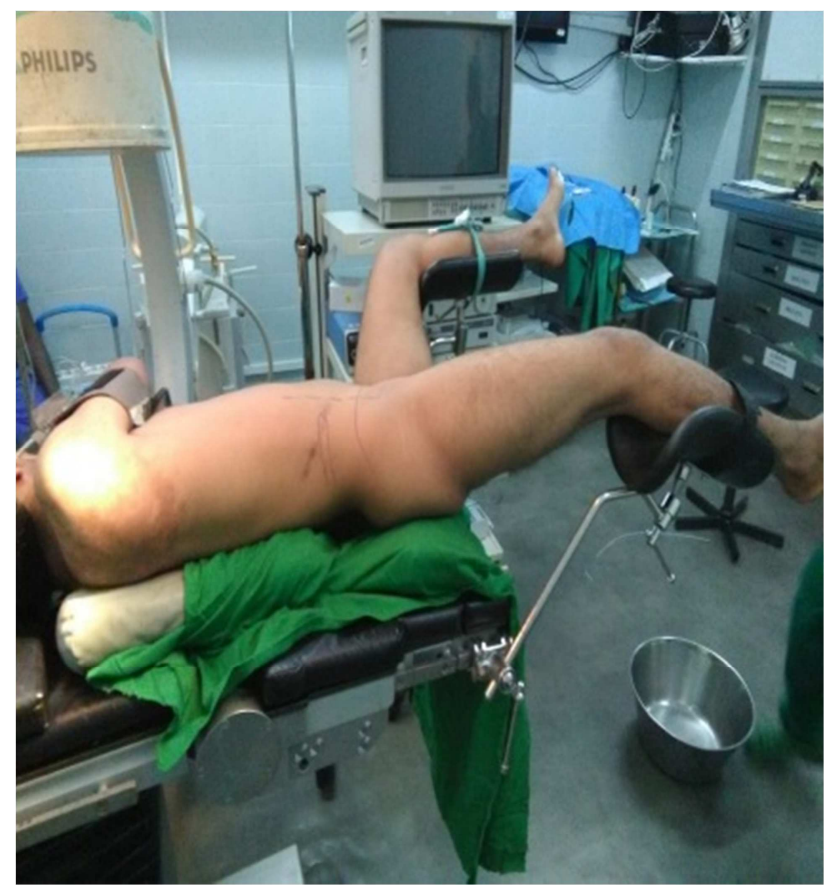

Figure 2. PCNL and ureteroscopy.

Figure 2 Under general ansthesia with endotracheal intubation, the patient was placed in the modified Valdivia position for simultaneous PCNL and ureteroscopy [7].

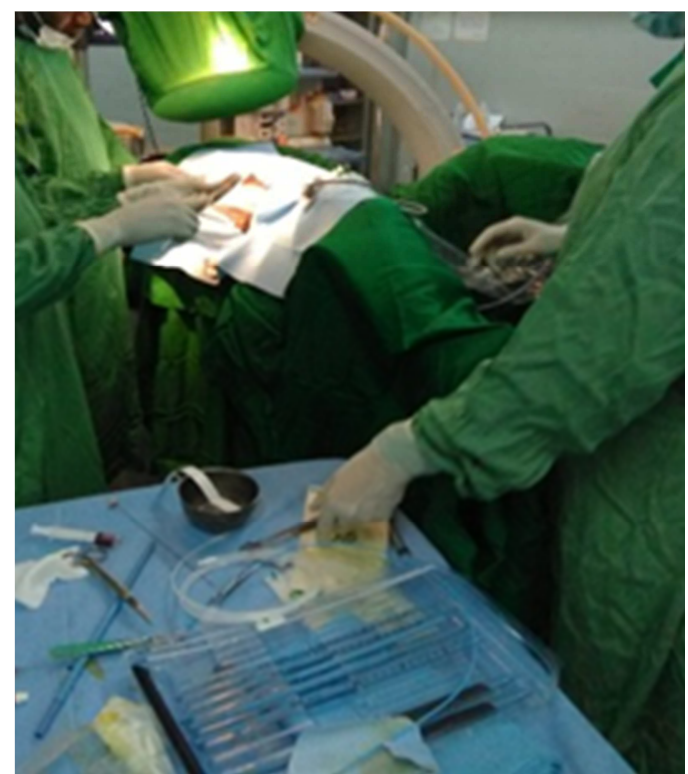

Figure 3. The patient was draped exposing the right flank with a C-arm fluoroscope set up.

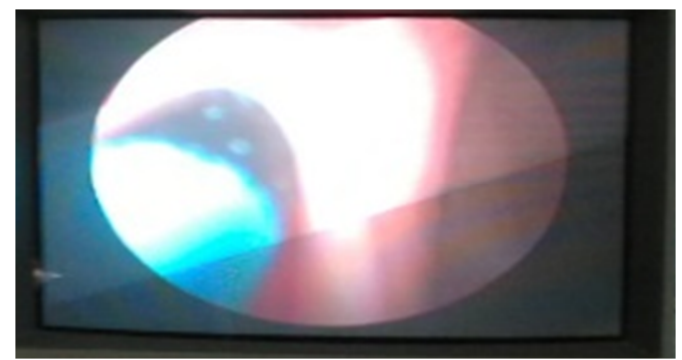

Figure 4. Ureteroscopy performed with left ureteral catheter placed for injection of methylene blue that better outlined the pelvicalyceal system.

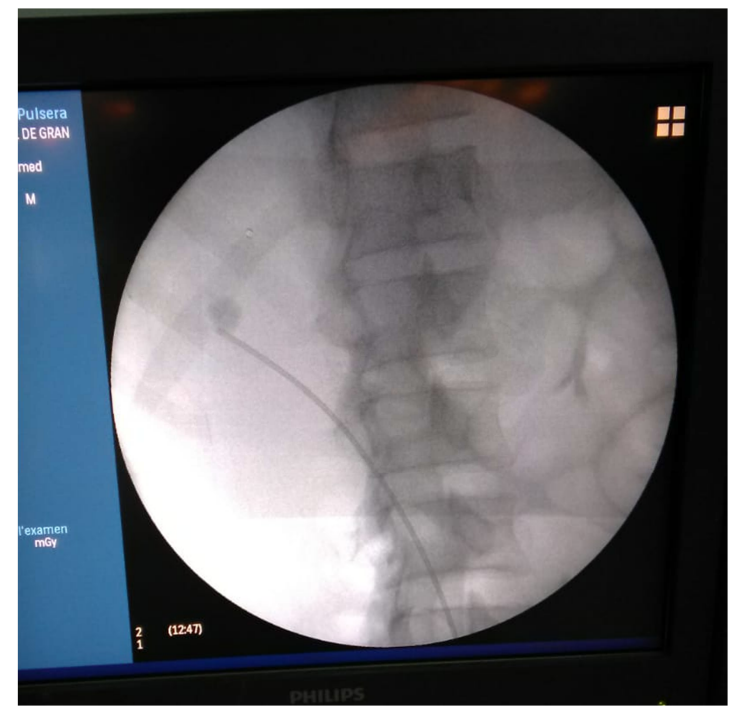

Figure 5. Initial puncture.

Figure 5 shows the Initial puncture performed with a $21 \mathrm{G}$ needle from below the 12 th rib, targeting the posterior calyx aligning the direction of access with the axis of the targeted calyx and an18G needle was inserted into the targeted calyx and a 0.038 -inch extra-stiff guidewire placed in pelvicalyceal system close to the calculi [8]. 


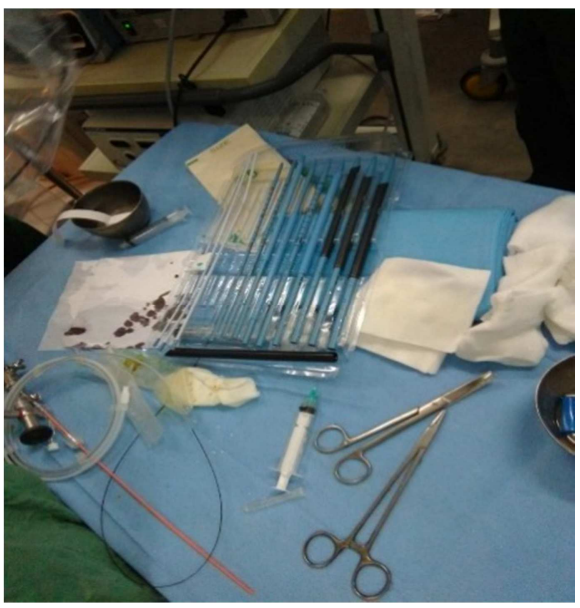

Figure 6. Serial dilatation with fascial dilators over the guidewire.

Figure 6 shows the serial dilatation with fascial dilators over the guidewire which was done to create a track into the collecting system under fluoroscopic guidance.

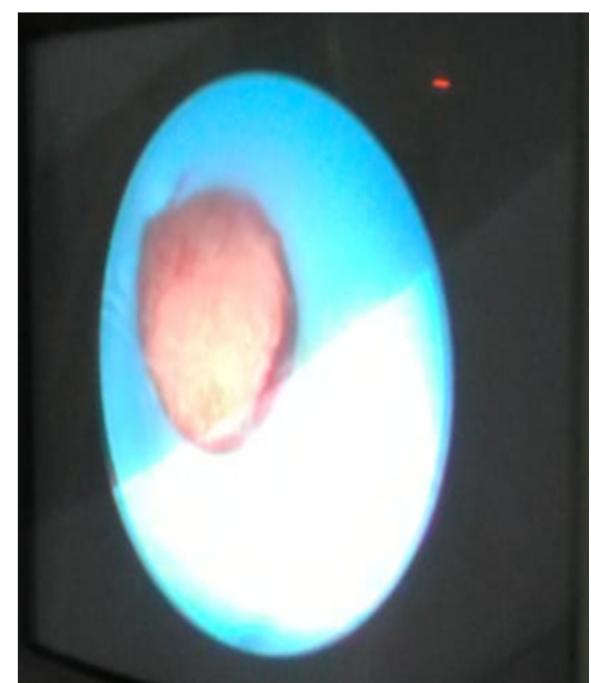

Figure 7. Amplatz sheath was slipped over the dilator and manipulated into the collecting system with the stone insight as shown [8].

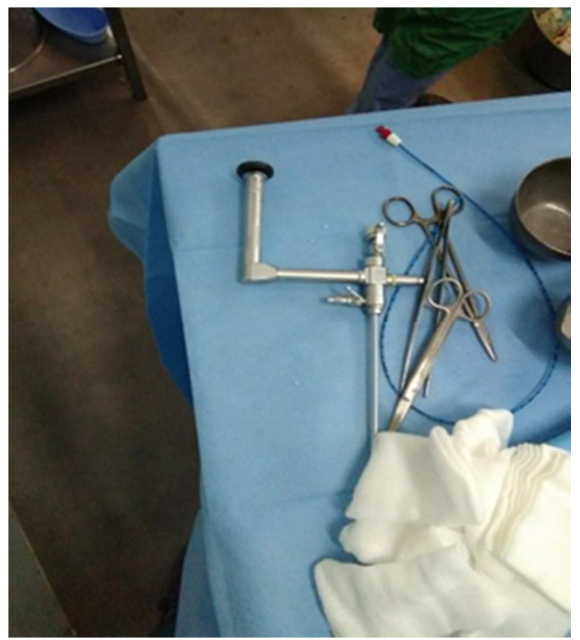

Figure 8. With the aid of an Amplatz sheath, the standard 26 Fr rigid nephroscope was placed into the renal collecting system to deal with the stones.

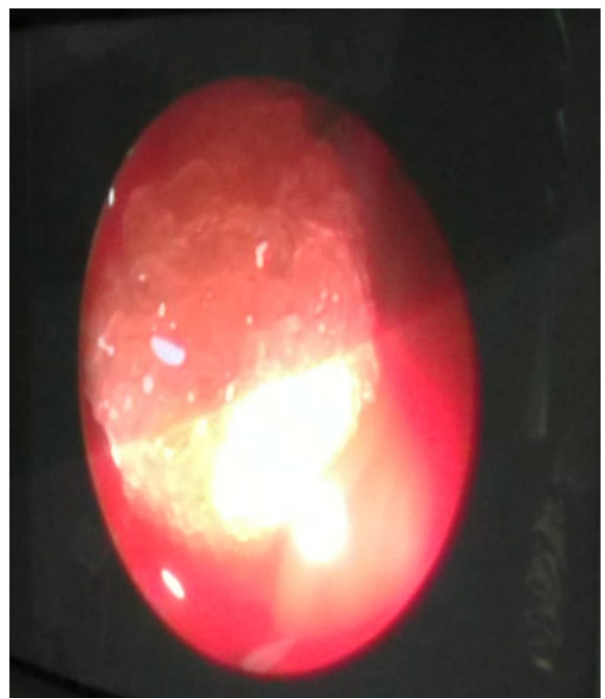

Figure 9. Ultrasonic lithotriptor was used to fragment the stone and particle irrigated with continuous warm normal saline.

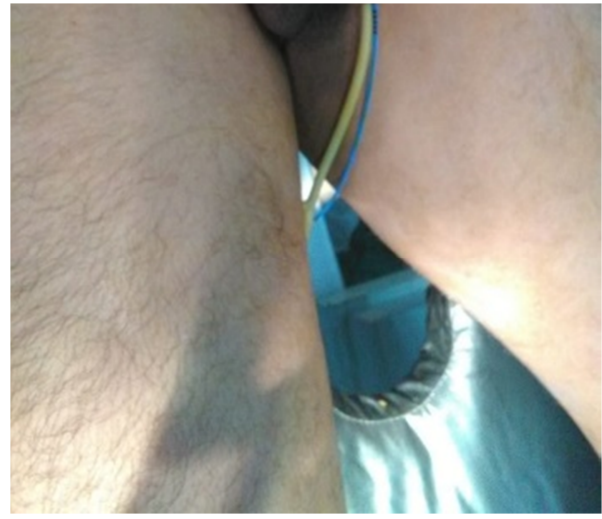

Figure 10. A urethral and ureteral catheter was left in place postoperatively for drainage.

\section{Discussion}

Surgical management of renal tract stone disease has evolved during the last two decades after the introduction of minimal invasive techniques, like ESWL and PCNL [1,9]. PCNL has become a common procedure performed in patients with renal calculi $[1,10]$.

Percutaneous nephrolithotomy (PNL) is recommended by the Guidelines of the European Association of Urology for the following indications [11]: Large stone burden $>2 \mathrm{~cm}$ or $1.5 \mathrm{~cm}$ for lower calyceal stones, staghorn stones, stones that are difficult to disintegrate by ESWL (calcium-oxalate monohydrate, brushite, cystine), stones refractory to ESWL or ureteroscopy, anatomic abnormalities, including horseshoe kidneys or ureteropelvic junction obstruction Stones within calyceal diverticula and obesity.

The preoperative evaluation involves a close study and analysis of imaging which includes a CT IVU [12]. The choice of puncture either fluoroscopic or ultrasound guided is dictated by the calyceal anatomy and the surgeon expertise in a technique [12].

Significant reductions in morbidity and cost have occurred 
because of the development of nephrostomy tract balloon dilators, improved grasping instruments, and the use of improved methods of stone fragmentation and removal [13]. The key to successful PNL is the adequate placement of the nephrostomy tract, thereby allowing appropriate access for complete stone removal [14]. The most important complication seen in PCNL surgery is bleeding, which can occur in forms of perioperative, immediate postoperative, and delayed [2]. The rate of blood transfusion is reported between $0-20 \%$ in the related studies [2]. The predicting factors for massive blood loss have been reported as body mass index, multiple punctures, dilation with larger dilators, stone size, long operative time, and the degree of preoperative hydronephrosis [15]. Mild fever post PCNL occurs in about one third of the patients, but incidence of sepsis is much lower, in patients treated with appropriate perioperative antibiotics [16]. Post-operative sepsis can be prevented by preoperative antibiotics, low-pressure irrigation, use of drainage when required [12].

\section{Conclusion}

PCNL has an essential role in managing patients with complex and large upper tract renal calculi. Its role in the management of renal calculi in sub-Saharan Africa is still evolving. It is time that urologist practicing in the subSaharan regions acquire the necessary skills in gaining percutaneous renal access if the equipments are available. With the latest advances in endourology, PCNL can provide optimal result with minimal patient morbidity.

\section{Conflict of Interest}

The authors declare no conflict of interest to this article.

\section{References}

[1] Reddy SV, Shaik AB. Outcome and complications of percutaneous nephrolithotomy as primary versus secondary procedure for renal calculi. Int Braz J Urol. 2016; 42 (2): 2629.

[2] Huseyin C, Cemal T, Ramazan A. AN OVERVIEW OF PERCUTANEOUS NEPHROLITHOTOMY: EMJ Urol. 2015; 3 [1]: 46-52.
[3] Nakada SY, Pearl MS. [CURRENT CLINICAL UROLOGY] ADVANCED ENDOUROLOGY. (C) 2006 Humana Press Inc. eISBN:1-59259-954-0.

[4] Al-Kohlany KM, Shokeir AA, Mosbah A, Mohsen T, Shoma AM, Eraky I, et al. Treatment of complete staghorn stones: a prospective randomized comparison of open surgery versus percutaneous nephrolithotomy. J Urol. 2005; 173: 469-73.

[5] Trinchieri A1, Ostini F,Nespoli R, Rovera F, Montanari E, Zanetti G. A prospective study of recurrence after a fist renal stone. J Endourol. 2006; 0: 17-20.

[6] Lojanapiwat B. Previous open nephrolithotomy: does it affect percutaneous nephrolithotomy techniques and outcome? J Endourol. 2006; 20: 17-20.

[7] Karaolides T, Moraitis K, Bach C, Masood J, Buchholz N. Positions for percutaneous nephrolithotomy: Thirty-five years of evolution. Arab J Urol. 2012; 10 (3): 307-16.

[8] Wong BTH. Percutaneous Nephrolithotomy. Medical Bulletin VOL.14 NO.10 OCTOBER 2009.

[9] Khan S, Toori LA, Anwer K. The effiacy of percutaneous nephrolithotomy in renal and upper uretric calculi. Pak J Med Res 2005; 44: 89-91.

[10] Srivastava A, Singh KJ, Suri A, Dubey D, Kumar A, Kapoor $\mathrm{R}$, et al. Vascular complications after percutaneous nephrolithotomy: are there any predictive factors? Urology. 2005; 66: 38-40.

[11] Tiselius HG, Ackermann D, Alken P, Buck C, Conort P, Gallucci M; Working Party on Lithiasis, European Association of Urology. Guidelines on urolithiasis. Eur Urol 2001; 40: $362-71$.

[12] Ganpule AP, Vijayakumar M, Malpani A, Desai MR. Percutaneou s nephrolithotomy (PCNL) a critical review: International Journal of Surgery Vol. 36 (2016); 660-664.

[13] Preminger MG, Clayman R, Curry T, Redman HC, Peters PC. Outpatient Percutaneous Nephrostolithotomy. The Journal of urology (1986). 136. 355-7. 10.1016/S0022-5347 (17) 448671.

[14] www.uptodate.com/ Options in the management of renal and ureteral stones in adults/ Glenn M Preminger, MD.

[15] Lee JK et al. Predictive factors for bleeding during percutaneous nephrolithotomy. Korean J Urol. 2013; 54 (7): $448-53$.

[16] Taylor E, Miller J, Chi T, Stoller ML. Complications associated with percutaneous nephrolithotomy. Transl Androl Urol. 2012; 1 (4): 223. 